CLINICAL STUDY

\title{
Anti-mullerian hormone is associated with advanced glycosylated end products in lean women with polycystic ovary syndrome
}

\author{
Evanthia Diamanti-Kandarakis, Athanasia Piouka ${ }^{1}$, Sarantis Livadas, Christine Piperi ${ }^{2}$, Ilias Katsikis ${ }^{1}$, \\ Athanasios G Papavassiliou ${ }^{2}$ and Demetrios Panidis ${ }^{1}$ \\ Endocrine Section, First Department of Medicine, University of Athens Medical School, Mikras Asias 75, Goudi 115-27, Athens, Greece, ${ }^{1}$ Division of \\ Endocrinology and Human Reproduction, Second Department of Obstetrics and Gynecology, Aristotle University of Thessaloniki, 119, Mitropoleos Street, \\ 54622 Thessaloniki, Greece and ${ }^{2}$ Laboratory of Biological Chemistry, University of Athens Medical School, Mikras Asias 75, Goudi 115-27, Athens, \\ Greece
}

(Correspondence should be addressed to E Diamanti-Kandarakis; Email: akandara@otenet.gr)

\begin{abstract}
Objective: Oocyte maturation process characterizes polycystic ovary syndrome (PCOS). The mechanisms of this abnormality leading to chronic anovulation are under investigation. Advanced glycosylated end products (AGEs), a marker of oxidative stress linked with oocyte maturation are localized in granulosa cells and are increased in sera, in women with PCOS. The aim of this study was to investigate the relationship, whether there is an association between the anti-mullerian hormone $(\mathrm{AMH})$, a hormone produced by granulosa cells and AGEs in ovulatory and anovulatory PCOS (PCOS-Anov), as well as in non-PCOS anovulatory (Non-PCOS Anov) women.

Design: Cross-sectional study.

Methods: Data from sixty women with PCOS (37 anovulatory and 23 regularly ovulating) were compared with eleven Non-PCOS Anov women and 25 normal women. In each subject biochemical, hormonal, and ultrasonographic parameters were studied.

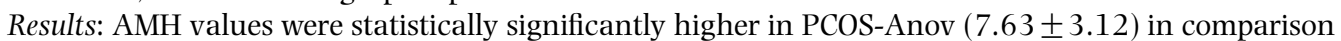
with ovulatory PCOS (PCOS-Ov; 4.92 \pm 2.50 ), Non-PCOS Anov (3.66 \pm 1.4 ), and controls $(4.02 \pm 1.27 \mathrm{ng} / \mathrm{ml})$. AGEs demonstrated a similar pattern: $8.70 \pm 1.65$ in PCOS-Anov, $7.43 \pm 1.79$, PCOS-Ov, 5.21 \pm 0.09 , Non-PCOS Anov, and 5.85 $\pm 0.89 \mathrm{U} / \mathrm{ml}$ in controls $(P<0.005$ for all comparison respectively). Follicle number was significantly higher in PCOS-Anov in comparison with other groups. A significant positive correlation between AMH and AGEs was observed ( $r: 0.326, P<0.01)$, and with the estimated AMH/AGEs ratio to follicle number $(r: 0.42, P: 0.0001)$ and the presence of anovulation. Conclusions: These data suggest that an oxidative marker, AGEs, and AMH, may interact in the anovulatory mechanisms in women with PCOS.
\end{abstract}

European Journal of Endocrinology 160 847-853

\section{Introduction}

Polycystic ovary syndrome (PCOS) is the most common endocrinopathy in women of reproductive age and is characterized by anovulation, hyperandrogenemia, and polycystic ovary morphology on ultrasound $(1,2)$ and different phenotypes appear to have different metabolic load (3). The pathophysiological mechanisms leading to anovulation in PCOS are under investigation $(4,5)$. The defective selection mechanism seems to lead in an accumulation of small antral follicles, which contributes significantly to the production of anti-mullerian hormone (AMH) (6).

$\mathrm{AMH}$ is a member of the transforming growth factor ß family, which is synthesized by granulosa cells of primary follicles and expressed in preantral and small antral follicles (4 mm) (7). In PCOS, the follicular excess is mainly due to an increase of small antral follicles ranged from 2 to $5 \mathrm{~mm}$ in size (8). Therefore, elevated AMH levels have been demonstrated in PCOS women from several investigators, suggesting that raised $\mathrm{AMH}$ levels could represent an indicator of disturbed ovulatory process in PCOS (9-13). AMH has an inhibitory effect on FSH sensitivity (14), explaining in part the mechanism via which AMH interferes with anovulation.

Recent data have shown that oxidative stress is involved in the pathophysiology of anovulation (15-17). However, clinical studies linking oxidative stress and anovulation in PCOS are lacking, although from in vitro studies oxidative stress has been shown to be involved in the pathogenesis of insulin resistance and hyperandrogenism in PCOS (18-20). Oxidative stress 
represented by elevated serum levels of advanced glycosylated end products (AGEs) and increased expression of their multiple ligand receptor on macrophages in PCOS have been demonstrated recently. AGEs that are products of non-enzymatic glycation and oxidation of proteins and lipids, have been found to induce oxidative stress and conversely oxidative stress stimulates AGEs formation (21-23). AGEs have been found to be elevated in different nosological entities including insulin and non-insulin resistant women with PCOS (24-26). In fact, it has been shown from our group that AGEs and their receptor (RAGE) have been localized by immunohistochemistry with significantly higher intensity on granulosa cells obtained from PCOS compared with controls (27). Moreover, in animals fed with AGE-enriched diet, significant elevation of serum AGEs and androgens levels, associated with increased deposition of AGEs in the ovarian tissues resulted (28). Taking into account the above data, an interaction of androgens and AGEs could be linked in the pathophysiology of ovarian dysfunction in PCOS.

In the present study, the potential relationship between AGEs, as mediators of oxidative stress immunonolocalized in the granulosa cells of polycystic ovarian tissue was investigated with $\mathrm{AMH}$, a hormone derived from granulosa cells. For this purpose, 96 women were studied. Specifically, hormonal and ultrasound data from a cohort of 60 women suffering from PCOS (37 anovulatory and 23 regularly ovulating) were compared with 11 anovulatory non-PCOS (NonPCOS Anov) women, and 25 normal women who served as controls. The studied groups were matched for age and body mass index (BMI).

\section{Subjects and methods}

\section{Subjects}

The study group comprised women who were presented to the outpatient endocrine clinic due to menstrual irregularities. The diagnosis of PCOS was based on Rotterdam Consensus criteria (29). Hyperandrogenemia was assessed as total testosterone levels above the 95th percentile of the levels detected in the group of normal menstruating women. Other androgen excess disorders (congenital adrenal hyperplasia) were excluded accordingly. A synacthen test was conducted in each woman with a basal 17-hydroxyprogesterone (17OHP) plasma level $>1.5 \mathrm{ng} / \mathrm{ml}$.

Sixty women with PCOS, further divided into two subgroups according to their ovulation status were studied. The anovulatory PCOS (PCOS-Anov) group was characterized as 37 women with documented hyperandrogenemia, anovulation (low progesterone levels on three consecutive cycles) and polycystic ovarian morphology on ultrasound, and exclusion of other reasons of anovulation such as hyperpolactinemia, hypothyroidism, diabetes mellitus, systemic diseases, and disturbances of hypothalamic-pituitary-adrenal axis. The ovulating (PCOS-Ov) group was characterized as 23 women with documented ovulation (progesterone levels on three consecutive cycles $>5 \mathrm{mg} / \mathrm{dl}$ ), hyperandrogenemia and polycystic ovarian morphology on ultrasound. Four subjects from each group were on contraceptive pill therapy until 3 months before the study. It must be mentioned that the study of PCOS patients at least 3 months post pill constitutes a universally accepted approach. Moreover, since progesterone levels were estimated at three consecutive cycles in all subjects and the classification to ovulatory and anovulatory was based on these levels, one can be almost certain that the classification was the best that could be done on a clinical basis.

A third group of Non-PCOS Anov women was comprised from 11 women with anovulation. Namely, these patients suffered from exercise-associated anovulation $(n=4)$, non-classical CAH $(n=4)$, prolactinoma $(n=2)$, and eating disorders $(n=2)$. It is known that Non-PCOS Anov comprises a very heterogeneous group and patients with prolactinemia, NC-CAH, or anorexia are classified as Non-PCOS Anov women (30).

Finally, 25 healthy women with regular periods and no hyperandrogenemia, hirsutism or acne served as control group (C). All patients were euthyroid, normoandrogenemic, normoprolactinemic, and none has $17 \mathrm{OHP}>1.5 \mathrm{ng} / \mathrm{ml}$. Exclusion criteria for the study included age over 40 years, known cardiovascular disease, neoplasms, current smoking, diabetes mellitus, renal impairment (serum creatinine $>120 \mu \mathrm{mol} / \mathrm{l}$ ), hypertension (blood pressure $>140 / 90 \mathrm{mmHg}$ ). The enrolled population was clinically healthy and not suffering from chronic or acute disease. Oral contraceptives or other drugs that could interfere with the hormonal and metabolic studies, if administrated, were discontinued for at least 3 months before the study.

The homeostasis assessment model (HOMA-IR) according to the formula: HOMA = fasting insulin $(\mathrm{miU} / \mathrm{ml}) \times$ fasting glucose $(\mathrm{mmol} / \mathrm{l}) / 22.5$ has been used as a marker of insulin resistance, and free androgen index (FAI) was calculated by the formula: $\mathrm{FAI}=(T$ in $\mathrm{nmol} / \mathrm{l} / \mathrm{sex}$ hormone-binding globulin $(\mathrm{SHBG})$ in $\mathrm{nmol} / \mathrm{l}) \times 100$.

The protocol was approved by the Institutional Review Committee of Hippocratio Hospital and Laiko General Hospital, and written informed consent was obtained from each subject before entry into the study.

\section{Assays}

Blood samples were collected from all patients and healthy controls between 08:00 and 10:00 h, after an overnight fast. All samples were obtained during the early follicular phase (day 2-4 from the first day of spontaneous bleeding episode) or at any time in anovulatory women with progesterone levels $<5 \mathrm{ng} / \mathrm{ml}$. 
They were centrifuged immediately, and serum was stored at $-80{ }^{\circ} \mathrm{C}$ until assayed for glucose, insulin, total testosterone, SHBG, androstenedione $(\Delta 4 \mathrm{~A}), \mathrm{LH}, \mathrm{FSH}$, 17-OHP, and DHEA-sulfate (DHEAS) as previously described (23-25). AGEs were measured by competitive AGE-ELISA. Just prior to assay, sera were thawed and diluted 1:5 with dilution buffer (PBS), 0.02\% Tween-20 and $1 \mathrm{mM}$ sodium azide $\left(\mathrm{NaN}_{3}\right)$. AGE measurements were performed at the Chemwell analyzer (Awareness, Palm City, Florida, USA). The competitive AGE-ELISA procedure was performed as previously described (31).

\section{Ultrasound assessment}

Transvaginal ultrasound scans of the ovaries were performed during follicular phase for the ovulatory group and at any point of time for unovulatory group confirmed with progesterone levels $<5 \mathrm{ng} / \mathrm{ml}$, by experienced sonographers in all the subjects who participated in the study (women with PCOS and controls). The presence of polycystic ovaries was diagnosed by the presence of 12 or more follicles in each ovary measuring 2-9 $\mathrm{mm}$ in diameter, and/or increased ovarian volume $\left(>10 \mathrm{~cm}^{3}\right)$. Ovarian volume was calculated by the following formula: $V=(\pi / 6) \times$ ( $D$ length $\times D$ width $\times D$ thickness), where $D$ is dimension. The mean ovarian volume for each participant was calculated by adding the volume of each ovary and dividing by 2. If characteristic ultrasound of PCO morphology, according to Rotterdam criteria, was found either on two ovaries or one ovary then the diagnosis of PCO morphology was established. Subjects with an ovarian cyst $>10 \mathrm{~mm}$ were excluded from the study.

\section{Statistical analysis}

All continuous variables showed normal distribution as it was documented by the use of the KolmogorovSmirnov test. Data are presented as means and S.D. Comparison of parameters between groups was performed using the one-way ANOVA model. Pairwise comparisons were performed using the Bonferroni test. Multiple regression analysis was used to examine the relationship of AGEs and AMH with all other variables. All tests are two-sided, statistical significance was set at $P<0.05$. All analyses were carried out using the statistical package SPSS version 13.00 (SPSS Inc., Chicago, IL, USA).

\section{Results}

Data from 37 PCOS-Anov women were compared with data derived from 23 ovulatory PCOS, 11 Non-PCOS Anov women and 25 regularly ovulating women (controls). The four groups did not differ in age $(24.08 \pm 4.5,25.76 \pm 5.56,27.45 \pm 6.72$, and
$26.02 \pm 4.99$ years respectively), BMI $(22.21 \pm 1.82$, $22.07 \pm 1.76,21.18 \pm 2.17$, and $22.35 \pm 1.50 \mathrm{~kg} / \mathrm{m}^{2}$ respectively) and waist-to-hip ratio (WHR; $0.75 \pm 0.05$, $0.73 \pm 0.04,0.77 \pm 0.04$, and $0.75 \pm 0.05$ respectively). AMH values were statistically significantly higher in PCOS-Anov $(7.63 \pm 3.12)$ in comparison with PCOS-Ov (4.92 \pm 2.50$)$, Non-PCOS Anov (3.66 \pm 1.4$)$, and controls $(4.02 \pm 1.27 \mathrm{ng} / \mathrm{ml})$.

AGEs values demonstrated a similar pattern: $8.70 \pm 1.65$ in PCOS-Anov, 7.43 \pm 1.79 , PCOS-Ov, $5.21 \pm 0.09$, Non-PCOS Anov, and 5.85 $\pm 0.89 \mathrm{U} / \mathrm{ml}$ in controls $(P<0.005$ for all comparison respectively; Figs 1 and 2). Follicle number was significantly higher in the PCOS anovulatory group in comparison with the other groups, whereas mean ovarian volume was significantly higher in PCOS-Anov in comparison with the Non-PCOS Anov and controls. No differences between groups were observed considering serum concentrations of LH, FSH, glucose, insulin, and the insulin resistance index HOMA-IR. Additionally, serum levels of testosterone, $\Delta 4 \mathrm{~A}$, and DHEAS were significantly higher in PCOS subjects in comparison with Non-PCOS Anov and controls. The results are analytically depicted on Table 1 .

Pearson analysis in the total group has shown a significant correlation of AGEs with AMH ( $r: 0.326$, $P<0.01$; Fig. 3a), testosterone ( $r: 0.492, P<0.01)$, SHBG $(r:-0.338, P<0.01)$, FAI $(r: 0.366, P<0.01)$, $\triangle 4 \mathrm{~A}(r: 0.348, P<0.01)$, and DHEAS $(r: 0.318$, $P<0.01)$.

Correspondingly, a significant correlation of $\mathrm{AMH}$ with testosterone $(r: 0.428, P<0.01)$, FAI $(r: 0.283$, $P<0.01), \Delta 4$ A ( $r: 0.342, P<0.01), 170 H P(r: 0.240$, $P<0.05)$, LH $(r: 0.505, P<0.01)$, FSH $(r:-0.202$, $P<0.05)$, age $(r:-0.228, P<0.05)$, and follicle number $(r: 0.472, P<0.01)$ was found. Since follicle number was different in four groups, the ratio of $\mathrm{AMH}$ against follicles and correspondingly of AGEs against

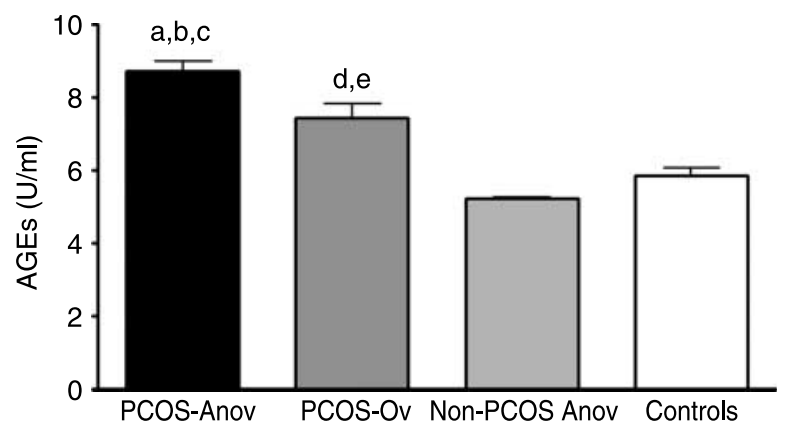

Figure 1 Serum levels of advanced glycosylated end products (AGEs) in the four groups. (a) Difference significant at the 0.01 level between PCOS-Anov and PCOS-Ov. (b) Difference significant at the 0.01 level between PCOS-Anov and Non-PCOS Anov.

(c) Difference significant at the 0.01 level between PCOS-Anov and controls. (d) Difference significant at the 0.01 level between PCOSOv and controls (e) Difference significant at the 0.01 level between PCOS-Ov and Non-PCOS Anov. 


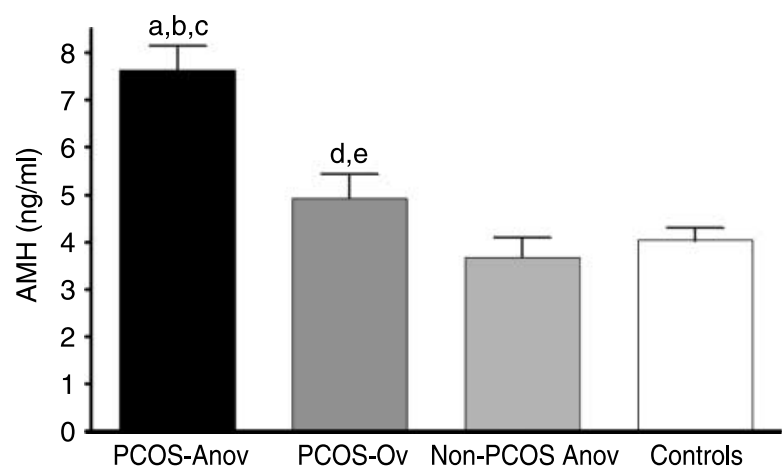

Figure 2 Serum levels of AMH in the four groups. (a) Difference significant at the 0.01 level between PCOS-Anov and PCOS-Ov. (b) Difference significant at the 0.01 level between PCOS-Anov and Non-PCOS Anov. (c) Difference significant at the 0.01 level between PCOS-Anov and controls. (d) Difference significant at the 0.01 level between PCOS-Ov and controls. (e) Difference significant at the 0.01 level between PCOS-Ov and Non-PCOS Anov.

follicles was estimated and a significant correlation between them was observed ( $r$ : 0.42, P: 0.0001, Fig. 3b). When multiple regression analysis was carried out in the total group using $\mathrm{AMH}$ as the dependent variable a significant correlation with gonadotropins $(P<0.0005)$, the existence of PCOS (P: 0.002) and anovulation ( $P$ : 0.028) was found. Correspondingly, when AGEs was used as the dependent variable, a significant correlation with BMI (P: 0.029), the existence of PCOS $(P<0.0005)$ and anovulation (P: 0.028) was found. Anovulation was highly correlated with either AMH (P: 0.032) and AGEs (P: 0.028).

\section{Discussion}

In the present study, it is demonstrated that anovulatory women with PCOS have higher serum levels of AMH, AGEs, androgens, and follicles number compared with Non-PCOS Anov women. These data support the presence of different pathophysiological mechanisms, between the two anovulatory groups. However, the fact that anovulatory women with PCOS and PCOS-Ov do not differ in androgens levels but still differ, at a statistically significant level, in AMH and AGEs levels as well as in follicle level number, suggests that additional factors interfere with maturation process in oocytes of PCOS women. The fact that these women do not differ in BMI, WHR, age, and insulin sensitivity, implies that these factors do not account for the observed differences in the studied groups.

Elevated serum AMH is considered to be linked with anovulation $(12,13,32,33)$. Furthermore, elegant studies have found that AMH production per granulosa cell was raised in PCOS in comparison with controls (11), and also increased expression of $\mathrm{AMH}$ and its receptor by granulosa cell from PCOS women (34). In addition, several research groups have shown that serum levels of AMH, in women with PCOS are two to threefold higher than in ovulatory women with normal ovaries $(10,35)$, an observation consistent with the findings of the present study.

Eldar-Geva et al. reported that AMH values were significantly increased in hyperandrogenic PCOS in comparison with normoandrogenic PCOS and controls (36). In the present study, the two anovulatory groups (PCOS \& Non-PCOS) that differ in androgen levels differ also in AMH concentrations, a finding in accordance with these data. Additionally, it has been shown that

Table 1 Pertinent data of the studied subjects.

\begin{tabular}{|c|c|c|c|c|}
\hline & PCos-Anov $(n=37)$ & PCOS-Ov $(n=23)$ & Non-PCOS Anov $(n=11)$ & Controls $(n=25)$ \\
\hline Age (years) & $24.08 \pm 4.5$ & $25.76 \pm 5.56$ & $27.45 \pm 6.72$ & $26.02 \pm 4.99$ \\
\hline BMI $\left(\mathrm{kg} / \mathrm{m}^{2}\right)$ & $22.21 \pm 1.82$ & $22.07 \pm 1.76$ & $21.18 \pm 2.17$ & $22.35 \pm 1.50$ \\
\hline WHR & $0.75 \pm 0.05$ & $0.73 \pm 0.04$ & $0.77 \pm 0.04$ & $0.75 \pm 0.05$ \\
\hline Testosterone (ng/dl) & $84.6 \pm 22.13^{\mathrm{a}, \mathrm{b}}$ & $83.34 \pm 22.82^{\mathrm{c}, \mathrm{d}}$ & $39.20 \pm 9.77$ & $40.14 \pm 11.51$ \\
\hline SHBG $(\mathrm{nmol} / \mathrm{l})$ & $44.69 \pm 17.42^{\mathrm{b}}$ & $46.6 \pm 22.3^{\mathrm{c}, \mathrm{d}}$ & $57.19 \pm 22.23$ & $69.84 \pm 21.93$ \\
\hline$\Delta 4 \mathrm{~A}(\mathrm{ng} / \mathrm{ml})$ & $2.98 \pm 1.07^{a, b}$ & $2.99 \pm 1.08^{\mathrm{c}, \mathrm{d}}$ & $1.83 \pm 0.43$ & $1.61 \pm 0.39$ \\
\hline FAl & $7.82 \pm 4.21^{\mathrm{a}, \mathrm{b}}$ & $7.91 \pm 4.62^{\mathrm{c}, \mathrm{d}}$ & $2.71 \pm 1.22$ & $2.23 \pm 1.04$ \\
\hline DHEAS (ng/ml) & $289.3 \pm 115.2^{a, b}$ & $315.9 \pm 147.4^{\mathrm{c}, \mathrm{d}}$ & $167.7 \pm 73.3$ & $190.7 \pm 78.5$ \\
\hline $170 \mathrm{HP}(\mathrm{ng} / \mathrm{ml})$ & $1.05 \pm 0.5^{\mathrm{b}}$ & $1.25 \pm 0.61^{\mathrm{c}}$ & $1.18 \pm 0.64$ & $0.69 \pm 0.39$ \\
\hline $\mathrm{LH}(\mathrm{IU} / \mathrm{I})$ & $8.65 \pm 6.02^{b}$ & $6.64 \pm 3.24$ & $6.08 \pm 3.58$ & $5.94 \pm 2.47$ \\
\hline FSH (IU/I) & $6.14 \pm 1.57$ & $6.32 \pm 2.01$ & $6.61 \pm 1.64$ & $6.53 \pm 1.77$ \\
\hline Glucose (mg/dl) & $92.91 \pm 12$ & $97.56 \pm 14$ & $97.09 \pm 16.42$ & $93.04 \pm 13.13$ \\
\hline Insulin $(\mu \mathrm{U} / \mathrm{ml})$ & $8.5 \pm 5.35$ & $8.13 \pm 4.93$ & $6.74 \pm 2.28$ & $6.90 \pm 3.46$ \\
\hline HOMA-IR & $1.98 \pm 1.47$ & $1.91 \pm 1.09$ & $1.63 \pm 0.74$ & $1.59 \pm 0.91$ \\
\hline Mean volume $\left(\mathrm{cm}^{3}\right)$ & $6.73 \pm 2.64^{a, b}$ & $6.81 \pm 2.23^{c, d}$ & $4.52 \pm 1.17$ & $5.02 \pm 2.14$ \\
\hline Follicle number & $19.43 \pm 7.71^{\mathrm{a}, \mathrm{b}, \mathrm{e}}$ & $12.79 \pm 3.95^{\mathrm{c}}$ & $12.05 \pm 2.98$ & $10.60 \pm 5.19$ \\
\hline
\end{tabular}

aDifference significant at the 0.01 level between PCOS-Anov and Non-PCOS Anov.

${ }^{b}$ Difference significant at the 0.01 level between PCOS-Anov and controls.

${ }^{\mathrm{C}}$ Difference significant at the 0.01 level between PCOS-Ov and controls.

${ }^{\mathrm{d} D i f f e r e n c e ~ s i g n i f i c a n t ~ a t ~ t h e ~} 0.01$ level between PCOS-Ov and Non-PCOS Anov.

eDifference significant at the 0.01 level between PCOS-Anov and PCOS-Ov. 

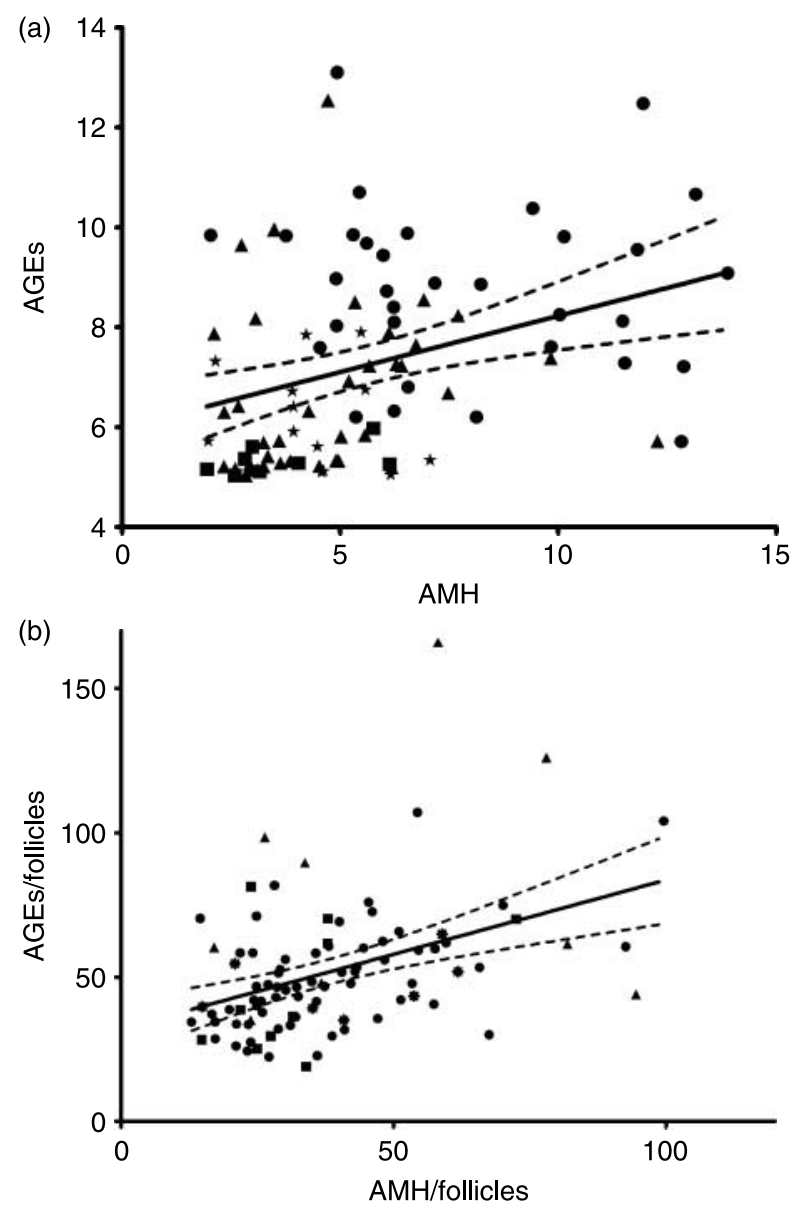

Figure 3 (a) Correlation of advanced glycosylated end products (AGEs) with AMH according to ovulation status ( $r .0 .326, P<0.01)$. (b) Correlation of advanced glycosylated end products (AGEs) ratio with follicles with $\mathrm{AMH} /$ follicles ratio according to ovulation status (r. 0.42, P: 0.0001). Circles denote PCOS-Anov, Triangles PCOS-Ov, Stars Non-PCOS Anov and Squares control respectively.

AMH by inhibiting aromatase activity leads in turn to increased local androgen concentrations (37-39). Experiments in rhesus monkeys have shown that androgens stimulate the initiation of primordial follicle growth and the proliferation of granulosa and theca cells of growing follicles reflected to AMH production (40). This observation is in support of our findings regarding the statistically significant increased number of follicles, AMH, and androgen levels in the PCOS-Anov compared with the Non-PCOS Anov. However, the fact that the two PCOS groups, anovulatory and ovulatory did differ in AMH levels, but not in the degree of hyperandrogenemia, implies that other factors in this case could also be involved.

It is of interest that the PCOS-Anov group has statistically significantly higher serum levels of AGEs compared with PCOS-Ov. This observation, in conjunction with the positive statistically significant correlation between AMH and AGEs, suggests an interaction of these molecules, highlighted after correction of both parameters for follicle number, which is different in the four groups and consequently could affect this relationship by mass effect of granulosa cells (Fig. 3). Further strengthening of a possible link of these molecules in the anovulatory process of PCOS, comes from the observation of higher expression and production of AMH and its receptor per granulosa cell in PCOS $(10,34)$, with higher immunohistochemical localization of AGEs and RAGE, as well as the nuclear localization of $\mathrm{NF} \kappa \mathrm{B}$ on the granulosa cells from human polycystic ovaries (27).

The mechanisms of interaction of AMH and AGEs in the oocyte maturation process in PCOS can not be elucidated in this study. However, the presented data are in support of the role of oxidative stress in ovulatory dysfunction $(41,42)$, since AGEs are well recognized mediators of increased oxidative stress $(43,44)$. It has been found that apoptosis in follicles is initiated by oxidative stress (45) and also that AGEs have been involved in the impaired folliculogenesis, (46) therefore linking these parameters with impaired follicle maturation. Antioxidant mechanisms have been localized in the granulosa and thecal cells of the growing follicle and consequently inadequate protection from oxidative stress could be a potential trigger for follicular atresia $(47,48)$. It is likely that increased oxidative stress, reflected by elevated serum AGEs in this study, may interfere in the ovulatory process, manifested by elevated AMH. From the above, the role of oxidative stress in anovulation appears to be supported, although the mechanisms by which oxidative stress interferes with oocyte maturation have not been clarified yet. Furthermore, well-designed studies have shown that oxidative stress modulates androgen production from theca cells of women with PCOS, both in vitro and in vivo $(18,42,49)$.

On the other hand, it is possible that AGEs, via increasing oxidative stress, could be linked with hyperandrogenemia, since oxidative stress has been shown to induce proliferation and growth of thecainterstitial cells on a dose-dependent manner (50). The positive correlation of AMH and AGEs levels between them as well as with androgens, shown in this study, suggests a role of hyperandrogenemia in $\mathrm{AMH}$ and/or AGEs levels. This possibility is further supported from the notion that the group of PCOS-Anov had significantly higher concentrations of AMH, AGEs, and androgens in comparison with Non-PCOS Anov group. Therefore, the interplay between AMH and AGEs could contribute to the anovulatory mechanisms via potentiation of hyperandrogenemia. Moreover, as it has been shown in regression analysis, either AMH or AGEs exhibit a strong relationship with anovulation, implying an interaction between them.

In conclusion, the data of this study demonstrate for the first time a link between AGEs, an oxidative stress marker distinctly elevated in women with PCOS, with 
AMH. Although causation cannot be deduced from the present study, this association between raised AMH and AGE levels implies an interrelated role to compromised follicle maturation process in PCOS. Clearly, further studies are required to explore the mechanisms of this potentially interactive relationship between oxidative mediators, anovulatory markers, and hyperandrogenemia in the dysfunctional maturation process of oocytes in patients with PCOS.

\section{Declaration of interest}

There is no conflict of interest that could be perceived as prejudicing the impartiality of the research reported.

\section{Funding}

This research did not receive any specific grant from any funding agency in the public, commercial or not-for-profit sector.

\section{References}

1 Dunaif A. Insulin resistance and the polycystic ovary syndrome: mechanism and implications for pathogenesis. Endocrine Reviews 199718 774-800.

2 Diamanti-Kandarakis E. Polycystic ovarian syndrome: pathophysiology, molecular aspects and clinical implications. Expert Reviews in Molecular Medicine $2008 \mathbf{1 0}$ e3.

3 Chang WY, Knochenhauer ES, Bartolucci AA \& Azziz R. Phenotypic spectrum of polycystic ovary syndrome: clinical and biochemical characterization of the three major clinical subgroups. Fertility and Sterility 200583 1717-1723.

4 Panidis DK, Rousso DH, Kourtis AI \& Papathanasiou KV. Could the theory of chaos contribute to the interpretation of pathogenesis of polycystic ovary syndrome? Clinical and Experimental Obstetrics and Gynecology $2003 \mathbf{3 0} 187-189$.

5 Franks S, Stark JE \& Hardy K. Follicle dynamics and anovulation in polycystic ovary syndrome. Human Reproduction Update 200814 367-378.

6 La Marca A, Orvieto R, Giulini S, Jasonni VM, Volpe A \& De Leo V. Mullerian inhibiting substance in women with polycystic ovary syndrome: relationship with hormonal and metabolic characteristics. Fertility and Sterility 200482 970-972.

7 Weenen C, Laven JS, Von Bergh AR, Cranfield M, Groome NP, Visser JA, Kramer P, Fauser BC \& Themmen AP. Anti-Müllerian hormone expression pattern in the human ovary: potential implications for initial and cyclic follicle recruitment. Molecular Human Reproduction 200410 77-83.

8 Pigny P, Jonard S, Robert Y \& Dewailly D. Serum anti-Mullerian hormone as a surrogate for antral follicle count for definition of the polycystic ovary syndrome. Journal of Clinical Endocrinology and Metabolism 200691 941-945.

9 Fallat ME, Siow Y, Marra M, Cook C \& Carrillo A. Mullerian inhibiting substance in follicular fluid and serum: a comparison of patients with tubal factor infertility, polycystic ovary syndrome, and endometriosis. Fertility and Sterility 199767 962-965.

10 Pigny P, Merlen E, Robert Y, Cortet-Rudelli C, Decanter C, Jonard S \& Dewailly D. Elevated serum level of anti-mullerian hormone in patients with polycystic ovary syndrome: relationship to the ovarian follicle excess and to the follicular arrest. Journal of Clinical Endocrinology and Metabolism $2003 \mathbf{8 8}$ 5957-5962.
11 Pellatt L, Hanna L, Brincat M, Galea R, Brain H, Whitehead S \& Mason H. Granulosa cell production of anti-Mullerian hormone is increased in polycystic ovaries. Journal of Clinical Endocrinology and Metabolism 200792 240-245.

12 Dewailly D, Catteau-Jonard S, Reyss AC, Maunoury-Lefebvre C, Poncelet E \& Pigny P. The excess in $2-5 \mathrm{~mm}$ follicles seen at ovarian ultrasonography is tightly associated to the follicular arrest of the polycystic ovary syndrome. Human Reproduction 2007 22 1562-1566.

13 Chen MJ, Yang WS, Chen CL, Wu MY, Yang YS \& Ho HN. The relationship between anti-Mullerian hormone, androgen and insulin resistance on the number of antral follicles in women with polycystic ovary syndrome. Human Reproduction 200823 952-957.

14 Durlinger AL, Gruijters MJ, Kramer P, Karels B, Kumar RT, Matzuk MM, Rose UM, de Jong FH, Uilenbroek JT, Grootegoed AJ \& Themmen AP. Anti-Mullerian hormone attenuates the effects of FSH on follicle development in the mouse ovary. Endocrinology 2001142 4891-4899.

15 Agarwal A, Gupta S \& Sharma RK. Role of oxidative stress in female reproduction. Reproductive Biology and Endocrinology 2005 328.

16 Tatone C, Carbone MC, Falone S, Aimola P, Giardinelli A, Caserta D, Marci R, Pandolfi A, Ragnelli AM \& Amicarelli F. Age-dependent changes in the expression of superoxide dismutases and catalase are associated with ultrastructural modifications in human granulosa cells. Molecular Human Reproduction 200612 655-660.

17 Fenkci V, Fenkci S, Yilmazer M \& Serteser M. Decreased total antioxidant status and increased oxidative stress in women with polycystic ovary syndrome may contribute to the risk of cardiovascular disease. Fertility and Sterility $2003 \mathbf{8 0}$ 123-127.

18 Kwintkiewicz J, Spaczynski RZ, Foyouzi N, Pehlivan T \& Duleba AJ. Insulin and oxidative stress modulate proliferation of rat ovarian theca-interstitial cells through diverse signal transduction pathways. Biology of Reproduction 200674 1034-1040.

19 Charitidou C, Farmakiotis D, Zournatzi V, Pidonia I, Pegiou T, Karamanis N, Hatzistilianou M, Katsikis I \& Panidis D. The administration of estrogens, combined with anti-androgens, has beneficial effects on the hormonal features and asymmetric dimethylarginine levels, in women with the polycystic ovary syndrome. Atherosclerosis $2008196958-965$.

20 Baynes JW. Role of oxidative stress in development of complications in diabetes. Diabetes $1991 \mathbf{4 0} 405-412$.

21 McCance DR, Dyer DG, Dunn JA, Bailie KE, Thorpe SR, Baynes JW \& Lyons TJ. Maillard reaction products and their relation to complications in insulin-dependent diabetes mellitus. Journal of Clinical Investigation 199391 2470-2478.

22 Singh R, Barden A, Mori T \& Beilin L. Advanced glycation end products: a review. Diabetologia 200144 129-146.

23 Diamanti-Kandarakis E, Piperi C, Kalofoutis A \& Creatsas G. Increased levels of serum advanced glycation end-products in women with polycystic ovary syndrome. Clinical Endocrinology $20056237-43$.

24 Diamanti-Kandarakis E, Katsikis I, Piperi C, Alexandraki K \& Panidis D. Effect of long-term orlistat treatment on serum levels of advanced glycation end-products in women with polycystic ovary syndrome. Clinical Endocrinology 200766 103-109.

25 Diamanti-Kandarakis E, Katsikis I, Piperi C, Kandaraki E, Piouka A, Papavassiliou AG \& Panidis D. Increased serum advanced glycation end products is a distinct finding in lean women with PCOS. Clinical Endocrinology 200869 634-641.

26 Peppa M, Uribarri J \& Vlassara H. Aging and glycoxidant stress. Hormones 20087 123-132.

27 Diamanti-Kandarakis E, Piperi C, Patsouris E, Korkolopoulou P, Panidis D, Pawelczyk L, Papavassiliou AG \& Duleba AJ. Immunohistochemical localization of advanced glycation end-products (AGEs) and their receptor (RAGE) in polycystic and normal ovaries. Histochemistry and Cell Biology 2007127 581-589. 
28 Diamanti-Kandarakis E, Piperi C, Korkolopoulou P, Kandaraki E, Levidou G, Papalois A, Patsouris E \& Papavassiliou AG. Accumulation of dietary glycotoxins in the reproductive system of normal female rats. Journal of Molecular Medicine 200785 1413-1420.

29 Rotterdam Consensus. Revised 2003 consensus on diagnostic criteria and long-term health risks related to polycystic ovary syndrome (PCOS). Human Reproduction 200419 41-47.

30 Hamilton-Fairley D \& Taylor A. Anovulation. BMJ 2003327 546-549.

31 Diamanti-Kandarakis E, Piperi C, Alexandraki K, Katsilambros N, Kouroupi E, Papailiou J, Lazaridis S, Koulouri E, Kandarakis HA, Douzinas EE, Creatsas G \& Kalofoutis A. Short-term effect of orlistat on dietary glycotoxins in healthy women and women with polycystic ovary syndrome. Metabolism $2006 \mathbf{5 5}$ 494-500.

32 Visser JA, de Jong FH, Laven JS \& Themmen APN. Anti-Mullerian hormone: a new marker for ovarian function. Reproduction 2006 131 1-9.

33 Chu MC, Carmina E, Wang J \& Lobo RA. Müllerian-inhibiting substance reflects ovarian findings in women with polycystic ovary syndrome better than does inhibin B. Fertility and Sterility 200584 1685-1688.

34 Catteau-Jonard S, Soazik PJ, Leclerc A, Gonzales J, Dewailly D \& di Clemente N. Anti-Mullerian hormone, its receptor, FSH receptor and androgen receptor genes are overexpressed by granulosa cells from stimulated follicles in women with polycystic ovary syndrome. Journal of Clinical Endocrinology and Metabolism 2008 $934456-4461$.

35 Laven JS, Mulders AG, Visser JA, Themmen APN, De Jong FH \& Fauser BC. Anti-Mullerian hormone serum concentrations in normoovulatory and anovulatory women of reproductive age. Journal of Clinical Endocrinology and Metabolism 200489 318-323.

36 Eldar-Geva T, Margalioth EJ, Gal M, Ben-Chetrit A, Algur N, Zylber-Haran E, Brooks B, Huerta M \& Spitz IM. Serum antiMullerian hormone levels during controlled ovarian hyperstimulation in women with polycystic ovaries with and without hyperandrogenism. Human Reproduction 200520 1814-1819.

37 di Clemente N, Goxe B, Remy JJ, Cate RL, Josso N, Vigier B \& Salesse R. Inhibitory effect of AMH upon aromatase activity and $\mathrm{LH}$ receptors of granulosa cells of rat and porcine immature ovaries. Endocrine 19942 553-558.

38 Grossman MP, Nakajima ST, Fallat ME \& Siow Y. Mullerianinhibiting substance inhibits cytochrome $\mathrm{P} 450$ aromatase activity in human granulosa lutein cell culture. Fertility and Sterility 2008 89 1364-1370.

39 Josso N, Racine C, di Clemente N, Rey R \& Xavier F. The role of antiMullerian hormone in gonadal development. Molecular and Cellular Endocrinology 1998145 3-7.
40 Vendola K, Zhou J, Wang J, Famuyiwa OA, Bievre M \& Bondy CA. Androgens promote oocyte insulin-like growth factor I expression and initiation of follicle development in the primate ovary. Biology of Reproduction 199961 353-357.

41 Agarwal A \& Allamaneni SS. Role of free radicals in female reproductive diseases and assisted reproduction. Reproductive Biomedicine Online 20049 338-347.

42 Gonzalez F, Rote NS, Minium J \& Kirwan JP. Reactive oxygen species-induced oxidative stress in the development of insulin resistance and hyperandrogenism in polycystic ovary syndrome. Journal of Clinical Endocrinology and Metabolism $200691336-340$

43 Basta G, Lazzerini G, Massaro M, Simoncini T, Tanganelli P, Fu C, Kislinger T, Stern DM, Schmidt AM \& De Caterina R. Advanced glycation end products activate endothelium through signaltransduction receptor RAGE: a mechanism for amplification of inflammatory responses. Circulation 2002105 816-822.

44 Devangelio E, Santilli F, Formoso G, Ferroni P, Bucciarelli L, Michetti N, Clissa C, Ciabattoni G, Consoli A \& Davì G. Soluble RAGE in type 2 diabetes: association with oxidative stress. Free Radical Biology and Medicine 200743 511-518.

45 Tsai-Turton M \& Luderer U. Opposing effects of glutathione depletion and follicle stimulating hormone on reactive oxygen species and apoptosis in cultured preovulatory rat follicles. Endocrinology 2006147 1224-1236.

46 Tatone C, Amicarelli F, Carbone MC, Monteleone P, Caserta D, Marci R, Artini PG, Piomboni P \& Focarelli R. Cellular and molecular aspects of ovarian follicle ageing. Human Reproduction Update 200814 131-142.

47 Lund SA, Murdoch J, Van Kirk EA \& Murdoch WJ. Mitogenic and antioxidant mechanisms of estradiol action in preovulatory ovine follicles: relevance to luteal function. Biology of Reproduction 1999 $61388-392$.

48 Valdez KE, Cuneo SP \& Turzillo AM. Regulation of apoptosis in the atresia of dominant bovine follicles of the first follicular wave following ovulation. Reproduction 2005130 71-81.

49 Sabuncu T, Vural H, Harma M \& Harma M. Oxidative stress in polycystic ovary syndrome and its contribution to the risk of cardiovascular disease. Clinical Biochemistry 200134 407-413.

50 Duleba AJ, Foyouzi N, Karaca M, Pehlivan T, Kwintkiewicz J \& Behrman HR. Proliferation of ovarian theca-interstitial cells is modulated by antioxidants and oxidative stress. Human Reproduction 200419 1519-1524.

Received 4 February 2009

Accepted 7 February 2009 